\title{
Psychosocial benefits of exercise for older adults with amnestic Mild Cognitive Impairment: Innovative practice
}

\author{
Juan Tortosa-Martínez \\ ${ }^{1}$ University of Alicante, Spain \\ Vicente J Beltrán-Carrillo \\ ${ }^{2}$ Miguel Hernández University, Spain \\ Nuria Caus \\ Marcos J Iglesias-Martínez \\ Inés Lozano-Cabezas \\ ${ }^{3}$ University of Alicante, Spain
}

Sandra Jimenez-Hernández

${ }^{4}$ Miguel Hernández University, Spain

JM Cortell-Tormo

\begin{abstract}
${ }^{5}$ University of Alicante, Spain
Juan Tortosa-Martínez, University of Alicante, Carretera San Vicente del Raspeig s/n, San Vicente del Raspeig 03690, Alicante, Spain. Email: juan.tortosa@gcloud.ua.es

The aim of this study was to explore the perceived psychosocial benefits of a three-month exercise program for 10 older adults with amnestic Mild Cognitive Impairment, a condition in which memory loss is the main symptom. Qualitative data were collected by observation (research diary) and 20 semi-structured interviews with the participants (10) and their caregivers (10). The narratives showed remarkable psychosocial benefits, such as improved mood, motivation, autonomy, perceived competence, self-esteem, and social relationships. The results of this study should provide new insights into the importance of exercise for this population, and may help to design appropriate programs for them.
\end{abstract}

Keywords: Mild Cognitive Impairment, physical activity, health-related quality of life, qualitative research, exercise

\section{Introduction}

Mild Cognitive Impairment (MCI) is considered as a clinical condition in which the person experiences memory loss to a greater extent to what it would normally be expected from normal aging, but not yet enough to meet criteria for a diagnosis of dementia or Alzheimer's disease (AD). MCI might be interpreted as an early stage of dementia, or as a separate condition (Peters \& Katz, 2015). Given the personal, familiar, and social impact of this disease, and without a known cure, it is imperative to design feasible strategies to improve the Health Related Quality of Life (HRQL) of people with MCI.AQ1 Exercise seems to be a promising strategy for improving physical function (such as cardiovascular capacity, strength, and balance), as well as cognitive function (such as executive function and memory) in people with MCI (Baker et al., 2010; Nagamatsu, Handy, Hsu, Voss, \& Liu-Ambrose, 2012). However, there are almost no studies focusing on the potential benefits of exercise for other relevant aspects of the HRQL of people with MCI such as emotional well-being or social aspects.

There is also a dearth of qualitative studies, which gives an incomplete picture of the possible benefits of exercise programs for this population. The use of a qualitative methodology could help to deepen the personal, subjective perceptions of people with MCI or dementia, bringing out the voice of these people and gaining a better understanding of what really matters to them (Cahill \& Díaz-Ponce, 2011). 
Thus, the aim of this study was to explore in depth the perceptions of a group of people with amnestic MCI, and their caregivers, in regard to the psychosocial benefits of a structured group-based exercise program.

\section{Methodology}

\section{Participants}

Potential participants were informed about the study by the staff of the Unit of Neurology of the Hospital of San Vicente del Raspeig, and provided with a written information sheet. General exclusion criteria included: living in a nursing home residence or attending a day-care center; non-compliance with testing procedures; physical, cardiovascular, or sensorial limitations for doing exercise safely; and severe apathy, delirium, or agitation. Ten patients diagnosed by trained neurologists with amnestic MCI according to Petersen (2004) criteria were recruited (Table 1). Amnestic MCI is a subtype of MCI, where memory loss is the main symptom.

Table 1. Sample characteristics.

\begin{tabular}{|l|l|}
\hline & a-MCI sample \\
\hline$N$ & 10 \\
\hline Age (years) & $74.40 \pm 8.3$ \\
\hline Gender & \\
\hline Women & $40 \%$ \\
\hline Men & $60 \%$ \\
\hline MMSE & $24.04( \pm 2.14)$ \\
\hline TMTA (seconds) & $75.28( \pm 23.82)$ \\
\hline TMTB (seconds) & $254.46( \pm 44.00)$ \\
\hline GDS & $3.46( \pm 2.16)$ \\
\hline 6MWT & $420.00( \pm 46.2)$ \\
\hline Systolic blood pressure & $130 \pm 12$ \\
\hline Diastolic blood pressure & $76 \pm 10$ \\
\hline GDS: Geriatic Depress &
\end{tabular}

GDS: Geriatric Depression Scale; MMSE: Mini Mental State Examination; 6MWT: Six-Minute Walk Test; TMTA: Trail Making Test form A; TMTB: Trail Making Test form B.

The perspectives of both patients and caregivers provide unique information about the patient's HRQL (Ready, Ott, \& Grace, 2004). Thus, 10 dyads consisting of people with MCI and their caregivers participated in the study.

All participants gave written informed consent to the protocol, which was approved by an ethics committee in the University of Alicante.

\section{The exercise program}

The exercise program was conducted for three months, three sessions per week, lasting 1 hour each session. Aerobic exercises were the main component of the program, and were performed at $60-75 \%$ of the maximum heart rate. Some light strength, balance, coordination, and flexibility exercises were also included. The program was conducted in groups, promoting social interaction.

\section{Qualitative data collection and analysis}

Different techniques were used to collect qualitative data. Two interviewers conducted and recorded a semi-structured interview with each of the 10 participants and their corresponding 10 caregivers ( 20 interviews), lasting from 30 minutes to 1 hour. Another researcher observed all the sessions of the exercise program and took field notes in a research diary.

The three qualitative researchers participating in the field work transcribed and analyzed the information that they had collected. Transcriptions were analyzed with the support of the software Aquad 7, which was used to organize and 
classify data efficiently. The analysis was carried out following the main strategies of a "conventional content analysis" (Hsieh \& Shannon, 2005).

\section{Results}

The results based in the perceptions of both participants and caregivers were divided into six main categories, related to the perceived psychosocial benefits of the program for participants with MCI.

\section{Enjoyment and improved mood}

Caregivers highlighted that the exercise program improved participants' mood, promoted enjoyment, and favored a more positive attitude among participants:

Very good, she would tell me all about it: "we were there throwing balloons to each other and we were laughing out loud, real laughter!" (Caregiver 3, husband)

I've felt mood improvements. I am in a very good mood ... I am very happy. (Participant 3, woman)

\section{Distraction from problems and the distress related to $\mathrm{MCl}$}

Participants reported that taking part in the exercise program was useful for them to avoid routine and boredom: "Last week when I didn't come (to the exercise program) I was bored" (Research diary, Participant 9, man). The exercise program kept their minds occupied in positives issues:

I would come here, do the things, and then I was not thinking about my problems... (Participant 3, woman)

The social relationships associated to the program also seemed to play an important role as a distraction and coping mechanism for dealing with the fact that they were probably going to develop AD:

The exercise was one thing, but what I enjoyed the most is that you talk, you get distracted for a while, and this way it seems that you are not always thinking about AD. (Participant 2, woman)

\section{Social relationships and support of the group}

In line with the previous section, the social relationships established in the exercise program were a source of benefits for the participants: "She feels like she is part of the group, and being recognized is very good for her" (Caregiver 7, husband). The program allowed participants to engage in a new social life. Meaningful social relationships emerged, along with strong group cohesion. These relationships played an important role for the perceived psychosocial benefits derived from the program:

We are already friends, I feel good here, and look ... I'm a very defensive person, but here I feel just great. (Research diary, Participant 4, man)

Logically, these good social relationships were a key component of the adherence to the exercise program: "Today I've come because I wanted to see you all" (Research diary, Participant 9, man).

\section{Enhanced self-esteem and perceived competence}

A perception of enhanced self-esteem and competence with the exercise program was apparent:

(Speaking about the exercise program) I would come out reinforced, it gave me life ... (Participant 6, man)

They are going to take us back home feeling like 18 years-old again. (Research diary, Participant 2, woman)

They were actually surprised about the new things they were able to do with "their age", such as using a stationary bicycle or elastic bands. Things which they thought were difficult to perform, but that they were actually able to carry out with competence:

Listen, there's people who can't do what we do! I've been going to gym (different program) for a long time, and when I tell people what we do here they don't believe me!!! (Research diary, Participant 2, woman)

Yesterday I took one of those camping mats and I stood up by myself. I don't even know how I did it!! (Research diary, Participant 1, woman) 


\section{Enhanced motivation}

The narratives of caregivers also stressed the relevance of the motivation that the program gave to participants. This motivation was two-fold. For some of them it was a motivation just to have something interesting to do during the day, something to look forward to with a positive attitude:

It has been very motivating for him, he knew he had to come, he had things to do. You would see him happier. (Caregiver 5, wife)

He doesn't remember anything, son. Sometimes, he'll repeat the same question so many times that he'll drive you crazy. He can't find a ring and he starts thinking that I've hidden it from him, then he'll drive me crazy asking me for the ring. However, he remembers perfectly the day and hours he has to come to the gym. It's something you have to live through to understand it. It's one of the very few things that's exciting for him. (Research diary, Caregiver 8, wife)

For others, the motivation was focused on the possibility of doing something to delay the onset or the development of the disease: "She is keen on using any possible means that are available for her to avoid developing that disease (Alzheimer's)" (Research diary, Caregiver 2, husband). The neurologists had told them that exercise could be good for them, which gave them hope. In the cases of those who were more aware of their condition and the likely future development of the disease, this became extremely important:

My mom (died from Alzheimer's) every time she moved you had to remain alert. One day I saw her almost drinking bleach... I don't want my children to go through all that with me, that's why I asked the doctor for something to delay the onset of the disease. (Participant 2, woman)

\section{Enhanced autonomy}

The exercise program also gave them the opportunity to show that they were still capable of doing something by themselves. This fact enhanced their autonomy perception. However, a clear conflict of some participants with their caregivers emerged when they reclaimed their autonomy and independence. This tension was manifested during the program, where they sometimes complained about the restrictions imposed by their family members for going to the program by themselves, or even being able to have lunch by themselves: "I don't want to be told what to do, I just want to feel alive" (Research diary, Participant 7, woman). Nevertheless, caregivers highly valued the fact that the exercise program had a positive effect in their relationship, because they thought participants were more relaxed and in a better mood.

\section{Discussion}

The exercise program provided participants with psychosocial benefits which positively influenced their HRQL, such as improved mood, motivation, autonomy, perceived competence, self-esteem, and social relationships. The only study that had previously reported psychosocial benefits is the one conducted by van Uffelen, Chin A Paw, Hopman-Rock, and van Mechelen (2007), where participants that achieved an attendance rate over $75 \%$ of the walking sessions over a one-year program, showed an improvement in their self-esteem and positive affect.

In our study, some psychosocial benefits derived from the exercise program could be interpreted from self-determination theory (Ryan \& Deci, 2000). The promoted activities and the social component of the exercise program satisfied participants' innate psychological needs of relatedness, competence, and autonomy. The participants felt part of a group and supported by their mates. They also felt competent with the new activities that they had learned and were able to do, and with their motor and physical improvements. Finally, they experienced autonomy because the program offered them the opportunity to do something by themselves, have their own social/leisure life and feel alive, distancing from dependence on others.

The satisfaction of these basic psychological needs fostered their well-being (feeling happier, improved mood, selfesteem, etc.), and promoted their self-regulated motivation (not imposed or controlled by others) to participate in the exercise program, keep a more active and social life, and cope with their condition with a more positive attitude. Their self-regulated motivation was also promoted the fact that they knew this activity was important to improve their HRQL, cope with MCI, and prevent or delay the development of AD. 
This last reason is in line with the postulates of the Common Sense Model of Illness Perception (Leventhal, Nerenz, \& Steele, 1984). According to this model, it is common that individuals with MCI initiate health behaviours, like practicing different kinds of physical activities, after their diagnosis and a perceived health threat, looking for adaptive responses to that threat (Morgan, Garand, \& Lingler, 2012). In this sense, the exercise program was considered by participants a coping strategy against MCI and AD. However, it is important to remark that the exercise program was also a coping strategy in another sense. It let participants have fun, feel the social support of their mates, and distract their mind from their problems and distress related to MCI and the possible development of AD. The important role that group-based exercise programs can play for coping with a chronic illness has already been reported (Beltrán-Carrillo, Tortosa-Martínez, Jennings, \& Sánchez, 2013).

People with MCI frequently report feeling sad and depressed because they are reliant on others and unable to do daily activities by themselves (Cahill \& Díaz-Ponce, 2011). With the shadow of AD over them, they may perceive the loss of autonomy as the beginning of feeling less as a human being, including the fear about losing completely their selves while they are still alive (Beard \& Neary, 2013). From this perspective, we can understand the conflicts we observed with the caregivers when the participants reclaimed their autonomy. Previous studies have highlighted the complicated situation lived by partners and relatives when they have to become a caregiver of a person with MIC or AD, giving rise to distress and ambiguous feelings and behaviours (Wegierek, 2012). Nevertheless, some caregivers highly valued the fact that the exercise program was having a positive effect in their relationship, because of the improved mood of the participants.

Last, the participants' adherence to the exercise program was a hopeful result, because it has been previously suggested that adherence to exercise interventions in people with MCI is difficult to achieve. Many barriers must be overcome, such as participants initial reluctance to start exercise, fear of injury or pain, chronic physical illness, lack of social support for exercise, or difficulties in learning and remembering new exercise routines (Logsdon, McCurry, Pike, $\&$ Teri, 2009). However, the participants of this study even showed their worry about the program ending, which is a good indicative about the feasibility of this type of programs for this population

\section{Limitations of the study}

The sample size of the study does not allow for generalizing results. The participants of the study were recruited from just one hospital (although by three different neurologists), which made some bias possible.

\section{Conclusion and practical implications}

The results of this study provide new insight about the importance of exercise for people with MCI, other than cognitive benefits. According to the findings of this research, taking part in a three-month group-based exercise program may report important psychosocial benefits for the HRQL of people with MCI. The public health systems should assume the responsibility to make exercise therapy available for this population.

In view of the findings of this study, future exercise programs for patients with MCI should consider the following:

- A positive social atmosphere in the exercise program is essential for the promotion of psychosocial benefits. The role of the instructors is crucial in this regard. The exercise programs should be directed by qualified sports sciences professionals with a specific training in MCI. The collaboration with practitioners and other health professionals should be desirable for the adequate design and implementation of high-quality exercise programs. Hospitals and sport centers should definitely be more interconnected.

- Instructors leading the exercise sessions should carry out strategies to promote perceived relatedness: (1) to show an affective attitude to participants, (2) to promote group-based exercises where members of the groups are frequently changed, (3) to value cooperation and respect among participants, and (4) to propose sometimes social activities outside the exercise program with both patients and caregivers, such as a meal or a trekking day trip involving short distances and light/moderate physical activity.

- Instructors should also carry out strategies to promote perceived competence: (1) to provide positive feedback focused on effort and personal improvement, (2) to propose tasks according to participants' level, (3) to provide enough time to learn and do activities with competence, and (4) to propose progressive learning processes (from less to more difficulty). 
- Instructors should also carry out strategies to promote perceived autonomy: (1) to let them choose some activities, material, or music, (2) to involve them in the leading of simple stretching exercises or the gathering of the material after the sessions, (3) to propose activities in which they can observe and correct the position or performance of one colleague, and (4) to provide them with practical guidelines and alternatives, so that they can do healthy exercise during their daily life, by themselves or with their caregivers.

- Finally, the instructor should educate participants in the benefits of exercise. Findings of this and other studies can be a resource to convince them about the importance of exercise for their HRQL. This fact could have an important effect on participants' adherence to exercise programs.

\section{Acknowledgments}

We thank neurologists M José Saénz, Inmaculada Abellán, and Gloria González for their valuable contribution to this work.

\section{Declaration of conflicting interests}

The author(s) declared no potential conflicts of interest with respect to the research, authorship, and/or publication of this article.

\section{Funding}

The author(s) received no financial support for the research, authorship, and/or publication of this article.

\section{References}

Baker, L. D., Frank, L. L., Foster-Schubert, K., Green, P. S., Wilkinson, C. W., McTiernan, A.Craft, S. (2010). Effects of aerobic exercise on mild cognitive impairment. Archives of Neurology, 67(1), 71-79.

Beard, R. L.Neary, T. M. (2013). Making sense of nonsense: Experiences of mild cognitive impairment. Sociology of Health and Illness, 35(1), 130-146. DOI:10.1111/j.1467-9566.2012.01481.x.

Beltrán-Carrillo, V. J., Tortosa-Martínez, J., Jennings, G.Sánchez, E. S. (2013). Contributions of a group-based exercise program for coping with fibromyalgia: A qualitative study giving voice to female patients. Women and Health, 53(6), 612-629.

Cahill, S.Díaz-Ponce, A. M. (2011). 'I hate having nobody here. I'd like to know where they all are': Can qualitative research detect differences in quality of life among nursing home residents with different levels of cognitive impairment? Aging \& Mental Health, 15(5), 562-572.

Hsieh, H. F.Shannon, S. E. (2005). Three approaches to qualitative content analysis. Qualitative Health Research, 15 (9), 1277-1288.

Leventhal, H., Nerenz, D. R.Steele, D. J. (1984). Illness representations and coping with health threats. In A. Baum, Taylor S. E.J. E. Singer (Eds.), Handbook of psychology and health: Social psychological aspects of health (pp. 219252). Hillsdale, NJ: Erlbaum.

Logsdon, R. G., McCurry, S. M., Pike, K. C.Teri, L. (2009). Making physical activity accessible to older adults with memory loss: A feasibility study. The Gerontologist, 49(1), 94-99. DOI:10.1093/geront/gnp082.

Morgan, G. H., Garand, L. I.Lingler, J. H. (2012). Self-initiated health behaviors following a diagnosis of mild cognitive impairment. Research in Gerontological Nursing, 5(2), 94-100. DOI:10.3928/19404921-20110831-02.

Nagamatsu, L. S., Handy, T., Hsu, L., Voss, M.Liu-Ambrose, T. (2012). Resistance training promotes cognitive and functional brain plasticity in seniors with probable mild cognitive impairment: A 6-month randomized controlled trial. Archives of Internal Medicine, 172(8), 666-668. DOI:10.1001/archinternmed.2012.379.

Peters, K. R.Katz, S. (2015). Voices from the field: Expert reflections on mild cognitive impairment. Dementia, 14(3) 285-297. 
Petersen, R. C. (2004). Mild cognitive impairment as a diagnostic entity. Journal of Internal Medicine, 256(3), 183194.

Ready, R. E., Ott, B. R.Grace, J. (2004). Patient versus informant perspectives of Quality of Life in Mild Cognitive Impairment and Alzheimer's disease. International Journal of Geriatric Psychiatry, 19, 256-265. DOI: 10.1002/gps. 1075.

Ryan, R. M.Deci, E. L. (2000). Self-determination theory and the facilitation of intrinsic motivation, social development, and well-being. American Psychologist, 55(1), 68-78.

van Uffelen, J. G. Z., Chin A Paw, M. J. M., Hopman-Rock, M.van Mechelen, W. (2007). The effect of walking and vitamin B supplementation on quality of life in community-dwelling adults with mild cognitive impairment: A randomized, controlled trial. Quality of Life Research, 16(7), 1137-1146.

Wegierek, A. M. (2012). Taking care of a loved one who has Alzheimer's disease. American Journal of Alzheimer's Disease and Other Dementias, 27(7), 463-467.

Juan Tortosa-Martínez obtained his PhD at the University of Alicante (Spain), where he has been a lecturer and a researcher in Physical Activity and Sports Sciences for 11 years. He is currently the Vice-Dean of the Faculty of Education, in charge of the Master's degrees and research of this faculty. His main focus of research is on physical activity and mental health, especially in older adults with cognitive impairment.

Vicente J Beltrán-Carrillo studied the Degree in Physical Activity and Sport Sciences and carried out his PhD dissertation at Universitat de València (Spain). He is currently a lecturer in the Sport Research Centre at Miguel Hernández University of Elche (Spain). His main interests are in behavioural aspects of physical inactivity, health, and well-being. He has published articles based in quantitative methods, although his main background and expertise is in qualitative research.

Nuria Caus obtained her PhD at the University of Valencia (Spain) in Physical Activity and Sport Sciences. She has been a lecturer and a researcher at the University of Alicante (Spain) for almost 10 years. Her main focus of research in on physical activity and health for people with special needs, including older adults with cognitive impairment.

Marcos J Iglesias-Martínez obtained his PhD hith honors in Educational Sciences at the University of Alicante-. Lecturer of the Department of General and Specific Didactics at the University of Alicante (Spain), where he is currently the academic secretary. His main focus and area of expertise is in qualitative research covering different topics such as gender studies or professional development in higher education. He has participated in several research projects related to these topics.AQ2

Inés Lozano-Cabezas obtained her PhD with honors in Educational Sciences. Lecturer of the Department of General and Specific Didactics at the University of Alicante (Spain), where she was the academic secretary. Her main focus and area of expertise is in qualitative research. She has participated in several research projects in this area.

Sandra Jimenez-Hernández studied the Degree in Physical Activity and Sport Sciences at the Universitat de València (Spain) and a Master's degree at Miguel Hernández University of Elche (Spain). She is currently a professional in the field of Physical Activity and Health.

JM Cortell-Tormo obtained his $\mathrm{PhD}$ at the University of Alicante (Spain), where he has been a lecturer and a researcher in Physical Activity and Sports Sciences for 12 years. He is currently the Vice-Dean of the Faculty of Education, in charge of the Physical Activity and Sports Science degree. His main focus of research is on physical activity and health. 


\section{Author Queries}

Query: AQ1 Please provide full form for ' $H R Q L$ '.

Response: Author query answered within text.

Query: AQ2 Please check edit: '...obtained his PhD with honors...'

Response: Author query answered within text. 